\title{
Investigation on a Novel Capacitive Electrode for Geophysical Surveys
}

\author{
Zhiyu Wang, ${ }^{1,2}$ Shun Wang, ${ }^{1}$ Guangyou Fang, ${ }^{3}$ and Qunying Zhang ${ }^{1}$ \\ ${ }^{1}$ Key Laboratory of Electromagnetic Radiation and Sensing Technology, Chinese Academy of Sciences, Beijing 100090, China \\ ${ }^{2}$ University of Chinese Academy of Sciences, Beijing 100149, China \\ ${ }^{3}$ Chinese Academy of Sciences, Beijing 100090, China
}

Correspondence should be addressed to Zhiyu Wang; wangzhiyul1@mails.ucas.ac.cn

Received 6 December 2015; Revised 29 May 2016; Accepted 31 May 2016

Academic Editor: Alberto J. Palma

Copyright (C) 2016 Zhiyu Wang et al. This is an open access article distributed under the Creative Commons Attribution License, which permits unrestricted use, distribution, and reproduction in any medium, provided the original work is properly cited.

\begin{abstract}
Nonpolarizable electrodes are applied widely in the electric field measurement for geophysical surveys. However, there are two major problems: (1) systematic errors caused by poor electrical contact in the high resistive terrains and (2) environmental damage associated with using nonpolarizable electrodes. A new alternative structure of capacitive electrode, which is capable of sensing surface potential through weak capacitive coupling, is presented to solve the above problems. A technique is introduced to neutralize distributed capacitance and input capacitance of the detection circuit. With the capacitance neutralization technique, the transmission coefficient of capacitive electrode remains stable when environmental conditions change. The simulation and field test results indicate that the new capacitive electrode has an operating bandwidth range from $0.1 \mathrm{~Hz}$ to $1 \mathrm{kHz}$. The capacitive electrodes have a good prospect of the applications in geophysical prospecting, especially in resistive terrains.
\end{abstract}

\section{Introduction}

Most electrical or electromagnetic geophysical methods, such as magnetotellurics, induced polarization, direct current (DC) resistivity imaging, and other new similar electromagnetic geophysical methods, need to acquire the direct current or alternating current electrical field signal when the system takes the measurement [1-5]. The measurement instrument always uses two separated grounded electrodes to detect the electric potentials which are different from them. The electric field intensity is calculated as electric potentials difference divided by the distance. In order to avoid the nature selfpolarization of the electrode while it is grounded or contracted to other conducted media, nonpolarizable electrode is applied widely in most electrical and electromagnetic geophysical instruments for measuring the electric field accurately [4]. The current typical nonpolarizable electrodes are $\mathrm{Pb}-\mathrm{PbCl}_{2}$ electrodes and $\mathrm{Ag}-\mathrm{AgCl}$ electrodes, and $\mathrm{Pb}$ $\mathrm{PbCl}_{2}$ electrodes are applied widely in onshore exploration. However, poor electrical contact is achieved in soil, under dry climatic conditions, or on rocky ground, which may lead to systematic errors [6]. Another problem of grounded electrode is that it is difficult to maintain the same contracted impedance between the electrode and the ground while the surrounded media take variety conductivity from point to point. So it affects the potential measurement accuracy and consistency. The damage and poison to the ground also are the problems of grounded electrodes.

In the 1970s, Timofeev et al. began to apply capacitive coupling to facilitate resistivity measurements on the frozen ground for the purpose of mapping permafrost $[7,8]$. They used the cables as transmitting electrode and receiving electrode. The displacement current for a certain frequency governed the energy transmission from the transmitter electrode to the ground, while the receiver electrode measured the surface potential [9]. As there is no galvanic current path between the cable and the ground, the method is named noncontacting capacitively coupled resistivity (CCR) or capacitive resistivity (CR). Correspondingly, the electrode is called capacitive electrode.

Since the 1990s, a mass of empirical, technical, and theoretical information had been researched, which was 
described by Kuras [10]. Kuras conducted a thorough study on the fundamental theory [8], giving an applicable frequency band for capacitive coupled resistivity:

$$
\frac{I}{2 \pi C U_{T}}<f<\frac{\rho}{\pi \mu_{0} L^{2}},
$$

where $f$ is the applicable frequency, $I$ is the introduced electric current, $C$ is the system capacitance, $U_{T}$ is the transmitter voltage, $\rho$ is the subsurface bulk resistivity, $\mu_{0}$ is the magnetic permeability, and $L$ is the transmitter-receiver separation. Formula (1) indicates that the low frequency is limited by the power output of the current systems and system capacitance.

In commercial applications, Geometrics Inc. and IRIS Instrument Inc. designed their instruments based on capacitive coupled resistivity: OhmMapper and CORIM. OhmMapper, which works at frequency of $16.5 \mathrm{kHz}$, is a capacitive coupled resistivity meter with line electrodes [11], while CORIM uses the plate-wire combination to couple to the ground, which works at frequency of $12 \mathrm{kHz}$ [12]. These systems are pulled along the ground either by a single person or by being attached to an all-terrain vehicle.

Compared with nonpolarizable electrodes, the utilization of the line electrodes or plate-wire electrodes speeds up the measurement and displays good applicability on highly resistive ground for near-surface investigations. However, there are some drawbacks. Firstly, the applicable frequency band limits the investigation depth [9]. Typically, investigation depth is in the range of $10 \mathrm{~m}$ to $20 \mathrm{~m}$. Secondly, the geometric factor of the CR method is approximated by the traditional four-point dipole-dipole geometry, but line electrode data do not fulfill the point source assumption of DC resistivity theory. In order to match the measured DC resistivity data, some CR models are developed to correct the geometrical factor and measurement biases $[9,13-16]$.

In the 2000s, GroundMetrics Inc. and QUASAR Federal Systems Inc. had developed a capacitive electrode named eQube, which operates at the frequency down to $0.1 \mathrm{~Hz}[17$, 18]. Similar to line electrode, eQube couples to the electric field via capacitive coupling directly. However, little data has been published on its performance in practical application.

The aim of this paper was to develop a new alternative capacitive electrode, which could be used for measuring the low-frequency geoelectric field accurately via weak capacitive coupling. A new structure of capacitive electrode, which is different from line electrode or plate-wire electrode, was developed. A metal plate is used for accumulating charges via capacitive coupling to the ground. The detection circuit is put closely to the plate, which converts charge into voltage and then outputs voltage signal. Because of the low output impedance, the signal can be transmitted for a long distance without the influences of the cable from the electrode to the data acquisition equipment. The main difference from line electrode is that the new capacitive electrode can be seen as point electrode, which is directly applied in the same geophysical measurement system without correcting effective dipole length. A technique is introduced to neutralize distributed capacitance and input capacitance of the detection circuit. With the capacitance neutralization technique, the transmission coefficient of capacitive electrode remains stable when environmental conditions change. The field test results indicate that the new capacitive electrodes have an operating bandwidth range from $0.1 \mathrm{~Hz}$ to $1 \mathrm{kHz}$ and they could measure the low-frequency geoelectric field accurately. The capacitive electrodes are well suited for current geophysical prospecting systems, such as transient electromagnetic system and audio magnetotelluric system. They have a good prospect of the applications in geophysical prospecting, especially in resistive terrains.

\section{Theory and Basic Principles}

The generic electrical field measurement system can be modeled as electrical circuit, where $Z_{\mathrm{P} 1}$ and $Z_{\mathrm{P} 2}$ are the contact impedance values of the electrodes and $Z_{\text {Earth }}$ is the ground impedance (see Figure 1(a)). There is a true current path for exchanging ions between the electrode and the solution, so the nonpolarizable electrodes or metal electrodes can be taken as resistance $R_{\mathrm{P}}$ (see Figure $1(\mathrm{~b})$ ). The capacitive electrodes, which couple to the electric field via capacitive coupling, can be taken as capacitance $C_{\mathrm{P}}$ as shown in Figure 1(c).

The paper assumes that electric potentials in the points of two electrodes are $\varphi_{1}$ and $\varphi_{2}$, and input impedance of the detection circuit is $Z_{\text {in }}$. The input voltage of the detection circuit with resistive electrodes is

$$
\Delta V_{R}=\frac{Z_{\mathrm{in}}}{R_{\mathrm{P} 1}+R_{\mathrm{P} 2}+Z_{\mathrm{in}}} \cdot\left(\varphi_{1}-\varphi_{2}\right) .
$$

As to capacitive electrodes, the input voltage of the detection circuit is

$$
\Delta V_{\mathrm{C}}=\frac{Z_{\mathrm{in}}}{1 / j \omega C_{\mathrm{P} 1}+1 / j \omega C_{\mathrm{P} 2}+Z_{\mathrm{in}}} \cdot\left(\varphi_{1}-\varphi_{2}\right) .
$$

Here, $\omega$ is angular frequency. When the operating conditions $Z_{\text {in }} \gg 1 / j \omega C_{\mathrm{P}}$ and $Z_{\text {in }} \gg R_{\mathrm{P}}$ are satisfied, $\Delta V_{C} \doteq \Delta V_{R}$ is set up. In contrast with nonpolarizable electrodes, capacitive electrodes can measure the geoelectric field more conveniently, which do not rely on exchanging ions between the electrode and the solution. This is important in the high resistive terrains, where it is hard to get good electrical contact. However, the value of $\Delta V_{C}$ is related to angular frequency $\omega$, and the impedance of capacitive electrode enlarges with the decrease of angular frequency. Because input impedance of the detection circuit is a finite value, there is a limitation of the lower frequency of capacitive electrode.

The capacitive electrode has two realization scenarios: the line electrode type and the plate-wire electrode type [8]. The line electrode type adopts a cable as electrode, which capacitively couples to the ground as shown in Figure 2(a). In the latter type, not only can the cable form a capacitor, but also the plate couples capacitively to the ground. Its capacitance is equal to the parallel combination values of the cable capacitance and the plate capacitance (Figure 2(b)). However, the effective dipole of those types of capacitive electrodes is not the geometric distance between the electrodes. But the effective dipole of the resistive electrodes is the geometric distance 


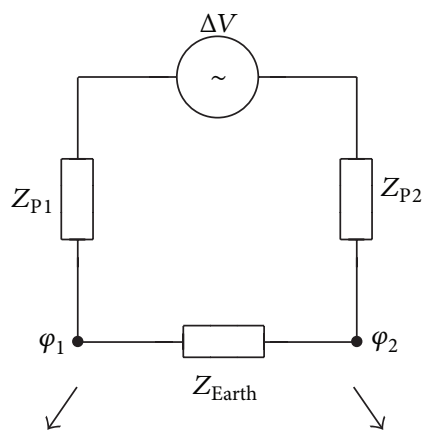

(a)

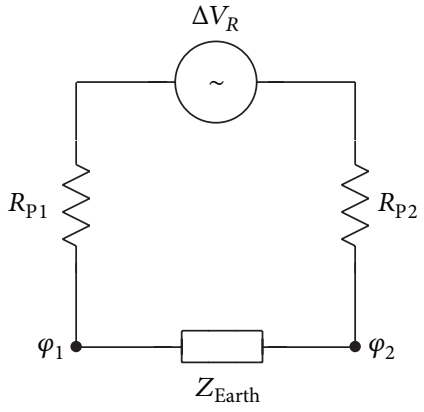

(b)

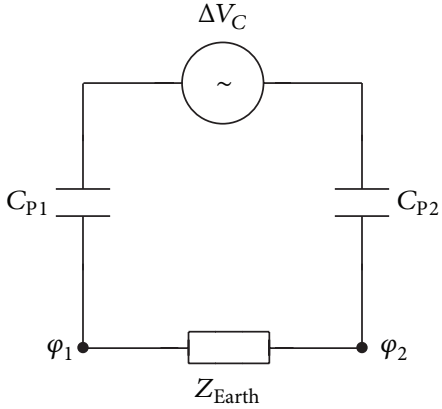

(c)

Figure 1: Equivalent electrical circuit models based on a modified form of Kuras et al. [8]. (a) A generic receiver system, (b) nonpolarizable electrodes, and (c) capacitive electrodes.

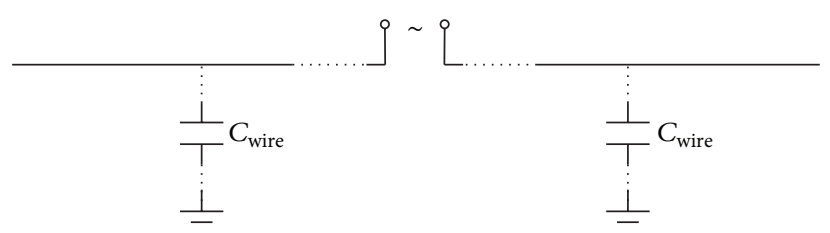

(a)

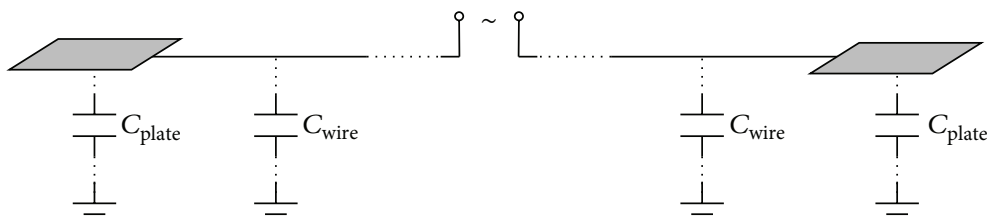

(b)

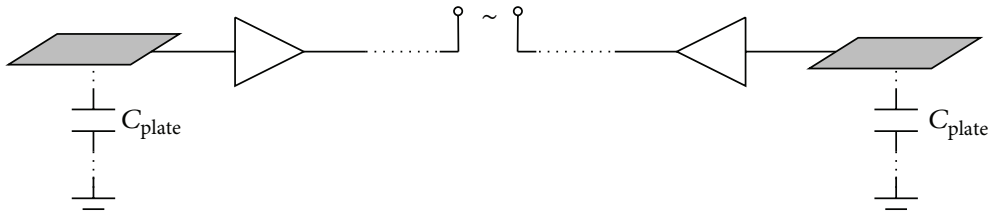

(c)

FIGURE 2: The conceptual model of (a) line electrode, (b) plate-wire electrode, and (c) new capacitive electrode.

between the electrodes. It will result in slight deviation between CR data and DC resistivity data. If the geometric distance increases to $100 \mathrm{~m}$ or more, which is common in DC resistivity method, the deviation is hard to be corrected.

As shown in Figure 2(c), the new capacitive electrode takes a novel structure: a metal plate is used as a receiver, which introduces charges via capacitive coupling to the ground. The detection circuit inside the capacitive electrode converts the charges into voltage, and then the voltage signal is transmitted through the cable. At last, it is acquired by data acquisition equipment. The equivalent model of the front detection circuit is shown in Figure 3, where $V_{s}$ is surface 


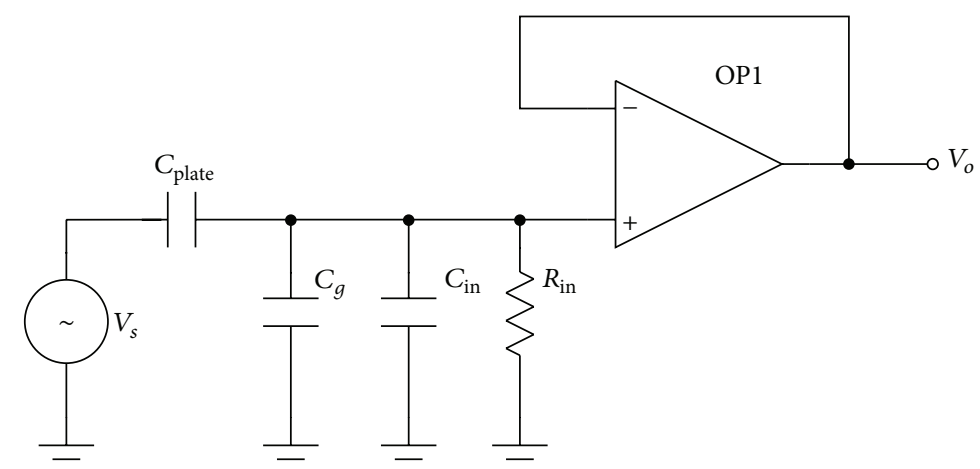

FIGURE 3: The equivalent model of front detection circuit.

potential, $C_{\text {plate }}$ is coupling capacitance between the electrode and the ground, $R_{\text {in }} / / C_{\text {in }}$ are input impedance of the operational amplifier OP1, and $C_{g}$ is the distributed capacitance from electrode to front detection circuit. The output voltage of OP1 can be expressed as

$$
V_{o}=\frac{s C_{\text {plate }} R_{\text {in }}}{1+s\left(C_{\text {plate }}+C_{g}+C_{\text {in }}\right) R_{\text {in }}} \cdot V_{s}
$$

where $s$ represents $j \omega$. In the regime where $s C_{\text {plate }} R_{\text {in }} \gg 1$, (3) can be simplified to the form of

$$
V_{o} \doteq \frac{C_{\text {plate }}}{C_{\text {plate }}+C_{g}+C_{\text {in }}} \cdot V_{s}
$$

For a rectangular plate of finite thickness suspended over the ground plane, an approximate functional expression of the capacitance is given as follows:

$$
\begin{aligned}
& C_{\text {plate }}=\varepsilon_{0} \varepsilon_{r} \cdot\left[1.15 \frac{w \cdot l}{h}+1.40\left(\frac{t}{h}\right)^{0.222} \cdot(2 w+2 l)\right. \\
& \left.\quad+4.12\left(\frac{t}{h}\right)^{0.728} \cdot h\right] .
\end{aligned}
$$

Here, $w, l$, and $t$ are the width, length, and thickness of the plate, respectively. $h$ is the height of the bottom face above the ground surface, $\varepsilon_{0}$ is absolute dielectric constant, and $\varepsilon_{r}$ is dielectric constant between the plate and the ground surface [8].

When $C_{\text {plate }} \gg C_{g}+C_{\text {in }}$, the output voltage $V_{o}$ is equal to the surface potential $V_{s}$. If the input impedance of the front detection circuit is large enough, the result is completely analogous to that of the nonpolarizable electrode. Compared with line electrode, the new capacitive electrode's coupling capacitance between the output cable and the ground does not contribute to the system capacitance any more. In other words, the new capacitive electrode can be viewed as a point electrode as nonpolarizable electrode. So it is suitable for current geophysical prospecting systems, such as transient electromagnetic system and audio magnetotelluric system. Of course, there is a limitation of bandwidth, which will be discussed in the next section.

\section{Design of Capacitive Electrode}

In a practice application, the dielectric constant of soil is not a constant with the changes of temperature and moisture content [19], which may lead to the change of coupling capacitance as shown in (5). Furthermore, the height from the ground surface to the bottom face of the plate and the flatness of the contact surface may also cause the coupling capacitance changing. In light of these problems, the charge amplifier, which is commonly used as the preamplifier of capacitive sensors such as pressure sensors and photoelectric sensors, is not suitable for capacitive electrode. Also, the circuit shown in Figure 3 is unpractical. There is no bias current path, which will result in the saturation of the amplifier and will make it unable to cope with the change of the coupling capacitance. Given these problems, a utility detection circuit is developed.

3.1. Design. To avoid the influence of the changes of the coupling capacitance, a front detection circuit is designed (see Figure 4). Here, $R_{\text {in }}$ and $C_{\text {in }}$ are input resistance and input capacitance of op-amp OP1. $V_{s}, C_{\text {plate}}$, and $C_{g}$ are surface potential, coupling capacitance between the electrode and the ground, and distributed capacitance, respectively. $C_{n}$, negative capacitance, is used to eliminate effects of input capacitance $C_{\text {in }}$ and distributed capacitance $C_{g}$. Parallel capacitance $C_{1}$ and feedback capacitance $C_{f}$ are used to adjust the cutoff frequency. In order to avoid the input bias current charging up the coupling capacitance and resulting in the saturation of the amplifier or producing a DC shift, a path for the input bias current to the amplifier is supplied by $R_{1}$ and $R_{2}$. The gain $A$ of operational amplifier OP2 is set by the ratio of $R_{3}$ and $R_{4}$, and $A=1+R_{3} / R_{4}$. The transfer function from the source to the output of the front detection circuit is expressed as

$$
\mathrm{TF}(s)=\frac{s R_{\mathrm{in}} n_{\mathrm{num}}+s^{2} R_{\mathrm{in}} m_{\mathrm{num}}}{R_{\mathrm{in}}+R_{1}+R_{2}+s\left[C_{1} R_{2}\left(R_{\mathrm{in}}+R_{2}\right)+C_{f} R_{1} R_{2}\right]+s R_{\mathrm{in}} n_{\mathrm{den}}+s^{2} R_{\mathrm{in}} m_{\mathrm{den}}},
$$




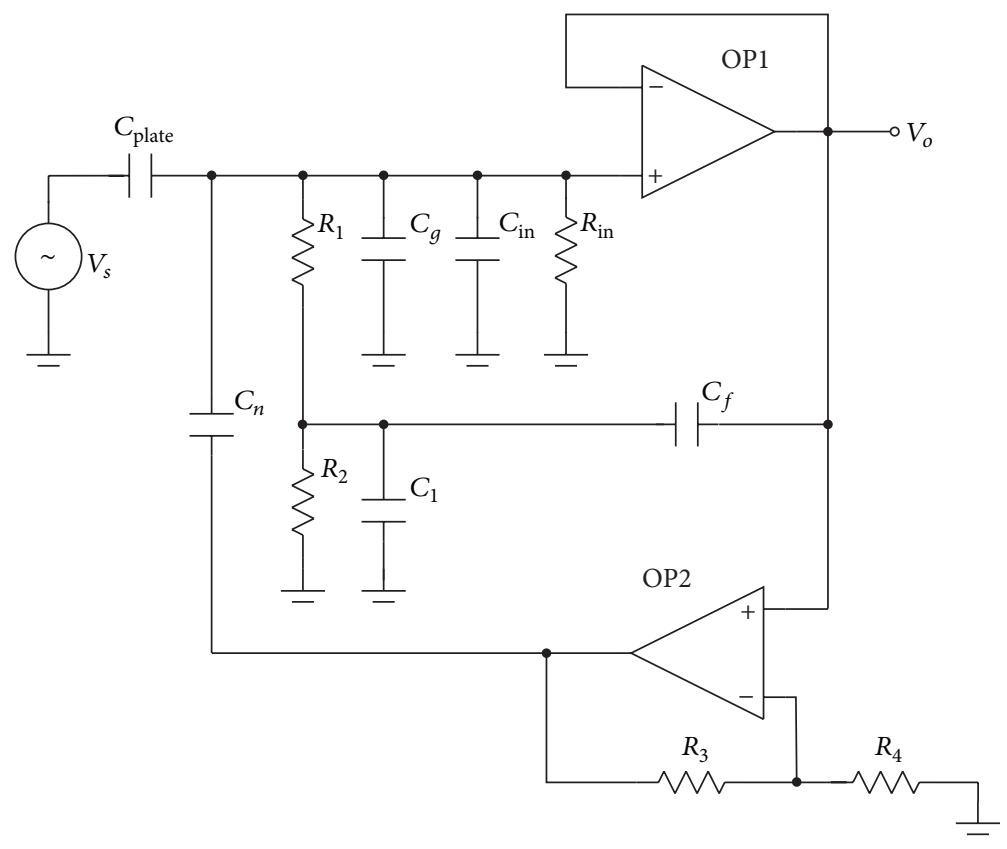

Figure 4: Practical front detection circuit.

where $m_{\text {num }}=\left(C_{1}+C_{f}\right) C_{\text {plate }} R_{1} R_{2}, m_{\text {den }}=\left(C_{1}+C_{f}\right)\left[C_{\text {plate }}+\right.$ $\left.C_{g}+C_{\text {in }}+(1-A) C_{n}\right] R_{1} R_{2}, n_{\text {num }}=C_{\text {plate }}\left(R_{1}+R_{2}\right)$, and $n_{\text {den }}=\left[C_{\text {plate }}+C_{g}+C_{\text {in }}+(1-A) C_{n}\right]\left(R_{1}+R_{2}\right)$.

In the regime where $R_{\text {in }} \gg R_{1}, R_{\text {in }} \gg R_{2}$, and $C_{g}+C_{\text {in }}=$ $-(1-A) C_{n}, m_{\text {num }}=m_{\text {den }}$ and $n_{\text {num }}=n_{\text {den }}$, and (6a) can be simplified to

$\mathrm{TF}(s)$

$$
=\frac{s n_{\text {num }}+s^{2} m_{\text {num }}}{1+s\left[C_{1} R_{2}+C_{f} R_{1} R_{2} / R_{\text {in }}+n_{\text {num }}\right]+s^{2} m_{\text {num }}} .
$$

The transfer function indicates that the capacitive electrode can be seen as a combination of high pass filter (HPF) and band pass filter (BPF), which has a unit gain in the passband. Denote the center frequency of BPF by $f_{0}$, quality factor by $Q$, and upper cutoff frequency and lower cutoff frequency by $f_{H}$ and $f_{L}$, respectively; then $f_{0}, Q, f_{H}$, and $f_{L}$ can be expressed as

$$
\begin{aligned}
f_{0} & =\frac{1}{2 \pi \sqrt{\left(C_{1}+C_{f}\right) C_{\text {plate }} R_{1} R_{2}}}, \\
Q & =\frac{\sqrt{\left(C_{1}+C_{f}\right) C_{\text {plate }} R_{1} R_{2}}}{\left[C_{1} R_{2}+C_{f} R_{1} R_{2} / R_{\text {in }}+C_{\text {plate }}\left(R_{1}+R_{2}\right)\right]}, \\
f_{H} & =\frac{f_{0}}{2}\left(\sqrt{4+\frac{1}{Q^{2}}}+\frac{1}{Q}\right) \\
f_{L} & =\frac{f_{0}}{2}\left(\sqrt{4+\frac{1}{Q^{2}}}-\frac{1}{Q}\right) .
\end{aligned}
$$

The cutoff frequency of HPF is the same as the center frequency of BPF. Hence, the cutoff frequency of the filter is dependent on coupling capacitance, feedback capacitance, and correlative resistances. It is easy to set the lower cutoff frequency by adjusting the feedback capacitance and resistances.

In (6a), the coefficient of quadratic term in the denominator is related to the input capacitance $C_{\text {in }}$ and distributed capacitance $C_{g}$. There will be an error in the output voltage if input capacitance and distributed capacitance change. Setting $C_{n}, C_{g}+C_{\text {in }}=-(1-A) C_{n}$, the coefficient of quadratic term in the denominator will be irrelevant to $C_{\text {in }}$ and $C_{g}$, referring to (6b). The coefficients of quadratic term in the numerator and denominator are the same, which means that the transmission coefficient is one even though the condition $C_{\text {plate }} \gg$ $C_{g}+C_{\text {in }}$ is not satisfied. It is fairly practical to deal with the variation of the coupling capacitance. However, input capacitance and distributed capacitance may not be constant during the life of capacitive electrode. Some steps should be taken to reduce the changes of the input capacitance and distributed capacitance, such as shortening the lead from the electrode to the input of front detect circuit as much as possible and fixing the lead to avoid it loosing.

The resistor $R_{1}$ can be replaced by diode or FET, which provides a low leakage current when $\mathrm{PN}$ junction is reverse biased. Two series diodes connected in opposite direction will serve the purpose of a large resistance when the voltage across the diodes swings within the forward voltage and breakdown voltage [20]. Another high pass filter can be added after the op-amp OP1 to remove the low-frequency noise and drift.

3.2. Simulation. Considering practical applications, the width $w$, length $l$, and thickness $t$ of a plate are $20 \mathrm{~cm}, 20 \mathrm{~cm}$, and $0.1 \mathrm{~cm}$, respectively. Parameters of the detective circuit 
TABLE 1: Transmission coefficient changes with coupling capacitance at frequency of $0.1 \mathrm{~Hz}$.

\begin{tabular}{lcccc}
\hline Height $(\mathrm{cm})$ & Coupling capacitance $(\mathrm{pF})$ & $\begin{array}{c}\text { Transmission coefficient } \\
\left(\Delta\left(C_{\mathrm{in}}+C_{g}\right)=0 \% C_{n}\right)\end{array}$ & $\begin{array}{c}\text { Transmission coefficient } \\
\left(\Delta\left(C_{\text {in }}+C_{g}\right)=10 \% C_{n}\right)\end{array}$ & $\begin{array}{c}\text { Transmission coefficient } \\
\left(\Delta\left(C_{\text {in }}+C_{g}\right)=20 \% C_{n}\right)\end{array}$ \\
\hline 0.25 & 171.06 & $1.000(0 \%)$ & $0.997(0.3 \%)$ & $0.994(0.6 \%)$ \\
1 & 46.75 & $1.000(0 \%)$ & $0.989(1.1 \%)$ & $0.979(2.1 \%)$ \\
2.5 & 21.23 & $1.000(0 \%)$ & $0.977(2.3 \%)$ & $0.955(4.5 \%)$ \\
5 & 12.41 & $1.000(0 \%)$ & $0.961(3.9 \%)$ & $0.925(7.5 \%)$ \\
10 & 7.77 & $0.999(0.1 \%)$ & $0.939(6.1 \%)$ & $0.885(11.5 \%)$ \\
\hline
\end{tabular}

are chosen according to the transfer function in Section 3.1. Input resistance $R_{\text {in }}$ and input capacitance $C_{\text {in }}$ of the op-amp OP1 are $10^{14} \Omega$ and $1 \mathrm{pF}$, respectively. Resistances $R_{1}$ and $R_{2}$, respectively, are $20 \mathrm{G} \Omega$ and $10 \mathrm{G} \Omega$, parallel capacitance $C_{1}$ is $50 \mathrm{nF}$, and feedback capacitance $C_{f}$ is $10 \mu \mathrm{F}$. Distributed capacitance $C_{g}$ can be calculated by (4) given a different coupling capacitance $C_{\text {plate }}$ and the ratio of source voltage and output voltage can be tested. Distributed capacitance $C_{g}$ is $4 \mathrm{pF}$. Accordingly, feedback capacitance is set as $5 \mathrm{pF}$. To observe the influences of variations of coupling capacitance and distributed capacitance on the transmission coefficient at frequency of $0.1 \mathrm{~Hz}$, the height $h$ of the plane above the ground surface and the residual unneutralized capacitance $\Delta\left(C_{\text {in }}+C_{g}\right)$ are changed, respectively.

As shown in Table 1, the value ranges of the coupling capacitance are from about $50 \mathrm{pF}$ to $8 \mathrm{pF}$ when the height $h$, respectively, is $0.25,1,2.5,5$, and $10 \mathrm{~cm}$. With the help of the negative capacitance, the transmission coefficient of capacitive electrode changes by less than $0.1 \%$ when input capacitance and distributed capacitance are neutralized completely. However, the effect of negative capacitance drops down when distributed capacitance and input capacitance deviate from the original values. The transmission coefficient bias enlarges with the increasing of the residual unneutralized capacitance and the height. Compared with the change of the gap height, the change of the unneutralized capacitance has a greater impact on the transmission coefficient. For getting a reliable measurement result, the gap height should be less than $5 \mathrm{~cm}$ and the residual unneutralized capacitance should be not more than twenty percent of the negative capacitance, which are not hard to achieve in practical application.

The transmission coefficient is held constant which equals the ratio of $m_{\text {num }}$ and $m_{\text {den }}$ at higher frequency more than $0.1 \mathrm{~Hz}$. At lower frequency, the measurement result will deteriorate further to be not credible. The cutoff frequency of high pass filter (HPF) is set to about $0.03 \mathrm{~Hz}$. After HPF, the operating bandwidth ranges from $0.1 \mathrm{~Hz}$ to $1 \mathrm{kHz}$ for obtaining accuracy of more than $90 \%$ (see Figure 5).

\section{Experiment Details}

4.1. Ground Conditions. The experiment was carried out from October 10 to October 15, 2014, in the area of Wuerhe, Karamay, Xinjiang Autonomous Region, China. This region was chosen for its dry soil and hostile environment. The top layer of the soil was gravel with thickness of about $3 \mathrm{~cm}$ to $5 \mathrm{~cm}$, the second layer was sandy soil, and the lower layer was sandy soil mixed with salinity. Because the experiment compared the differences between capacitive electrodes signal and resistive electrodes signal, it was not concerned with the geological situation of the entire region. The ground surface was not flat because of the gravel. And the measurement result could verify the influences of the contact surface flatness.

4.2. Experiment Arrangement. The experiment is conducted with BGP Inc. exploration. BGP explored the area using timefrequency electromagnetic method (TFEM), which adopted the arrangement as in Figure 6. The perpendicular distance between current dipole line and survey line was $8 \mathrm{~km}$. There were 24 survey points evenly distributed along the survey line and the center distance of two adjacent points was $400 \mathrm{~m}$. Each point contained two nonpolarizable electrodes and one magnetometer. Only two pairs of capacitive electrodes were deployed close to the nonpolarizable $\mathrm{Pb}-\mathrm{PbCl}_{2}$ electrodes coming from BGP Inc., and both of their dipoles were spaced $100 \mathrm{~m}$ apart. If capacitive electrodes got the same measurement waveforms at the same point, we will consider that capacitive electrodes have the ability to substitute nonpolarizable electrodes directly with no correction of measurement data.

For reducing connection resistance, nonpolarizable electrodes were buried about $20 \mathrm{~cm}$ under the ground and watered with enough brine. The connection resistance was less than $200 \Omega$ during the experiments. The new capacitive electrodes were put on the ground surface directly as shown in Figure 7.

4.3. Experimental Results and Discussion. Figure 8 shows time domain waveforms and power spectral density curves of the measurement data of capacitive electrodes and nonpolarizable electrodes when the transmitted frequency is $2 \mathrm{~Hz}$. As a premise of discussion, the data of nonpolarizable electrodes is assumed to be accurate and correct. The time domain waveforms of the capacitive electrodes and nonpolarizable electrodes are almost coincident. Through more test data comparisons, the ability of capacitive electrodes can be tested. Referring to Figure 9, magnitude ratios and phase differences of capacitive electrodes and nonpolarizable electrodes are around one with fluctuation of less than $4 \%$ and less than 20 degrees when the frequency ranges from $0.1 \mathrm{~Hz}$ to $1 \mathrm{kHz}$, respectively. The largest bias of the magnitude appears at frequency of $0.125 \mathrm{~Hz}$. The magnitude ratio is 0.967 , and the bias is $3.3 \%$, which is allowed in the geoelectric field measurement. At lower frequency ranges, the amplitude bias is increased 


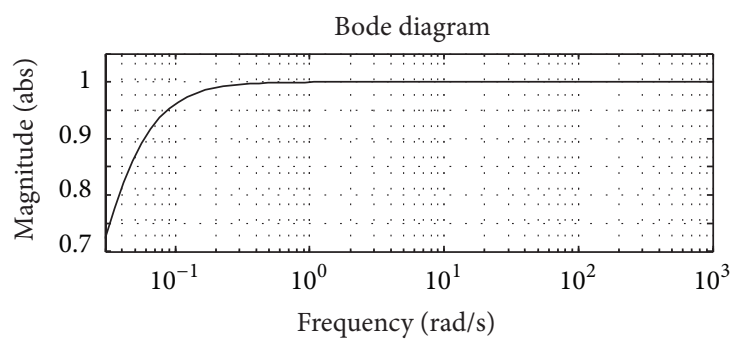

(a)

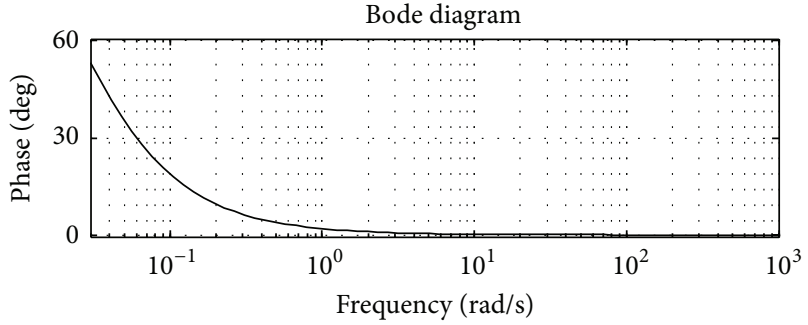

(b)

FIGURE 5: The frequency response curve of the new capacitive electrode.

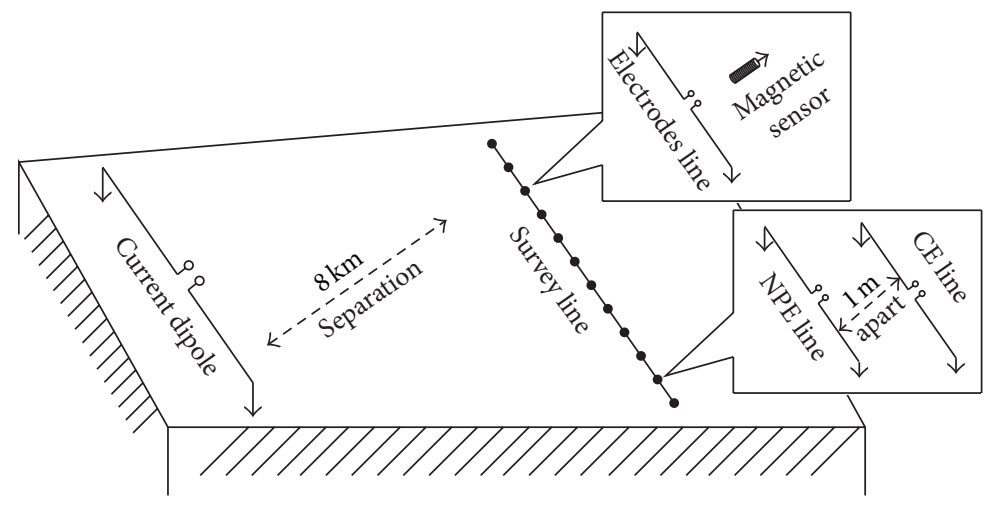

FIGURE 6: The experiment arrangement.

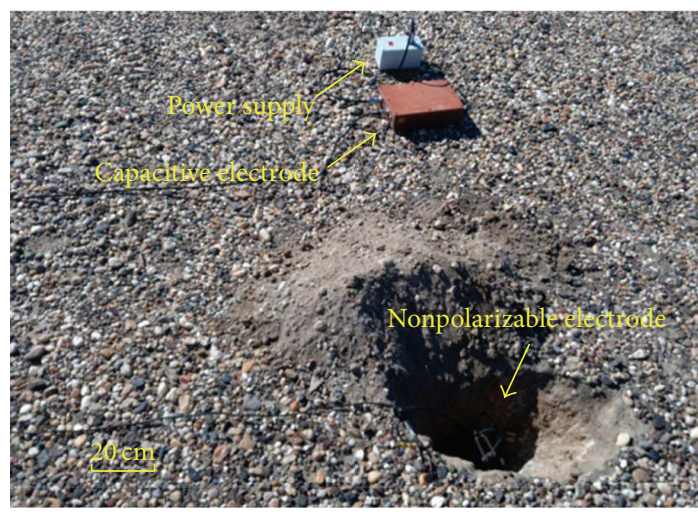

Figure 7: The electrodes at test site.

by about $25 \%$ at the roll-off frequency of $0.03 \mathrm{~Hz}$, which is less than the simulation results in Figure 5. It is considered that capacitive electrodes are capable of accurately measuring geoelectric field when the frequency ranges from $0.1 \mathrm{~Hz}$ to $1 \mathrm{kHz}$.

The background noises of nonpolarizable electrodes and capacitive electrodes, which include the intrinsic noise of electrodes and the noise of natural electric field, are about $0.5 \mu \mathrm{V} / \sqrt{\mathrm{Hz}}$ and $1.5 \mu \mathrm{V} / \sqrt{\mathrm{Hz}}$, respectively, at the frequency of $10 \mathrm{~Hz}$ (refer to Figure 8(b)). Because the electrodes were placed at the same point and the measurement data were recorded by the same equipment, the background noise differences of those two kinds of electrodes are caused by the electrode itself. The intrinsic noise of capacitive electrodes is about $1.5 \mu \mathrm{V} / \sqrt{\mathrm{Hz}}$ at the frequency of $10 \mathrm{~Hz}$. The noise is almost inversely proportional to frequency at lower frequency. At higher frequency range, the performances of capacitive electrodes and nonpolarizable electrodes are similar.

The new capacitive electrode combines the advantages of both the line electrode and the nonpolarizable electrode. Compared with the line electrode, the new capacitance electrode can measure the geoelectric field precisely down to $0.1 \mathrm{~Hz}$, which means that the system could have larger investigation depth. In contrast with nonpolarizable electrode, capacitive electrode can measure the geoelectric field without reliance on exchanging ions between the electrode and the solution. This is important in the high resistive terrains, where it is hard to get good electrical contact. The new capacitive electrode has the same measurement results as the nonpolarizable electrode with the same dipoles spacing. This indicates that the new capacitive electrode can be suitable for current geophysical system without correction of measurement biases.

\section{Conclusion}

This paper analyzes the theory of the new capacitive electrode and gives a practical detection circuit. Due to the variations of the dielectric constant of the soil and flatness between the electrode plate and ground surface or other factors, 


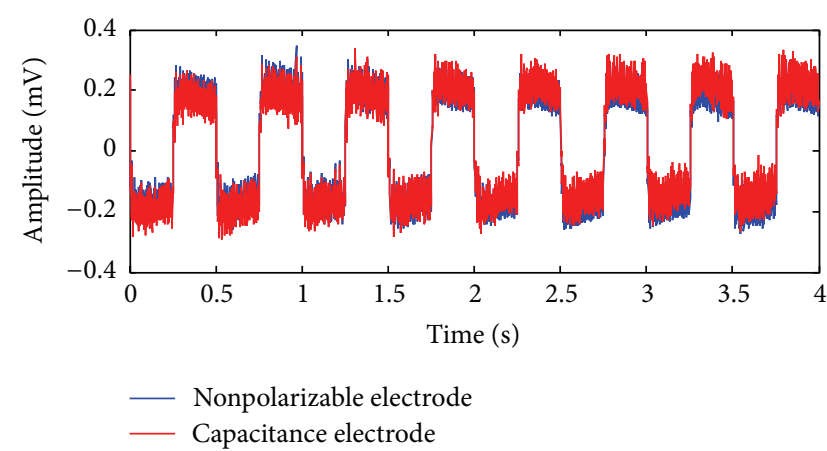

(a)

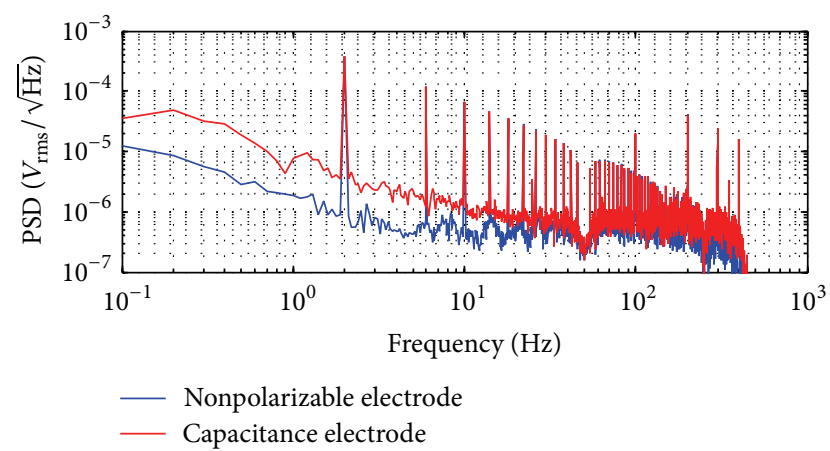

(b)

Figure 8: (a) Time domain waveform and (b) power spectral density curves of capacitive electrodes (red lines) and nonpolarizable electrodes (blue lines). The industry frequency $(50 \mathrm{~Hz})$ and its odd harmonics have been filtered out.

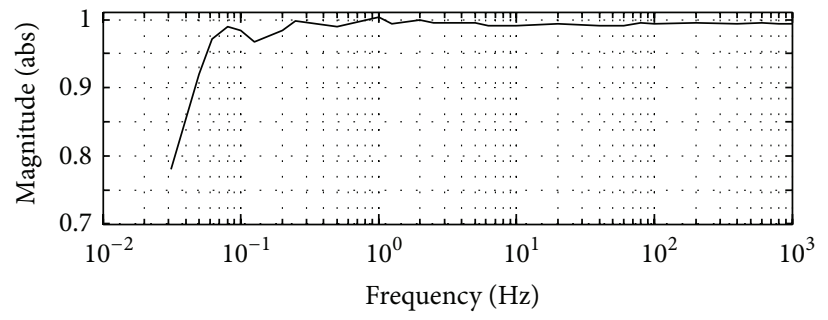

(a)

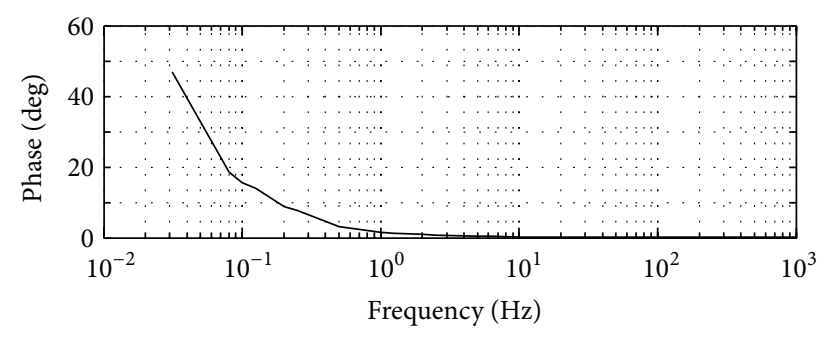

(b)

Figure 9: (a) Magnitude ratios and (b) phase differences of capacitive electrodes and nonpolarizable electrodes.

the coupling capacitance will change, which results in inaccurate data. The negative capacitance technique is applied to neutralize the input capacitance and stray capacitance, which is helpful for eliminating the deviation caused by this problem. In order to avoid the saturation in a pure capacitive measurement, a feedback loop is designed to provide a bias current path to ground without reducing the input impedance of the amplifier. The operating bandwidth ranges from $0.1 \mathrm{~Hz}$ to $1 \mathrm{kHz}$ via parameter matching between capacitive electrode and detection circuit. The power consumption of the detection circuit is less than $10 \mathrm{~mW}$.

The new capacitive electrodes respond directly to the electric field via capacitive coupling. Compared with the conventional nonpolarizable electrodes and other metal electrodes, they are easy to be used especially in the very resistive terrains, such as desert and permafrost. Unlike the conventional capacitive electrodes used in CR systems, the new capacitive electrodes can operate in a lower frequency range, which meets the requirements of most electrical or electromagnetic geophysical surveys methods. Furthermore, the new capacitive electrodes can be seen as point electrodes which can be applied directly in the existing electrical resistivity survey system with no correction in effective dipole length.

\section{Competing Interests}

The authors declare no competing interests regarding the publication of this paper.

\section{Acknowledgments}

This research was supported by R\&D of Key Instruments and Technologies for Deep Resources Prospecting, Grant no. ZDY2012-1-05. The authors are grateful to the help of BGP Inc., China National Petroleum Corporation, in the verification experiments in the area of Wuerhe in Xinjiang Autonomous Region.

\section{References}

[1] M. A. Goldstein and D. W. Strangway, "Audio-frequency magnetotellurics with a grounded electric dipole source," Geophysics, vol. 40, no. 4, pp. 669-683, 1975.

[2] T. Dahlin, "The development of DC resistivity imaging techniques," Computers and Geosciences, vol. 27, no. 9, pp. 1019-1029, 2001.

[3] W. M. Telford, L. P. Geldart, and R. E. Sheriff, Applied Geophysics, vol. 1, Cambridge University Press, Cambridge, UK, 1990.

[4] J. M. Reynolds, An Introduction to Applied and Environmental Geophysics, John Wiley \& Sons, New York, NY, USA, 2011.

[5] M. H. Loke, J. E. Chambers, D. F. Rucker, O. Kuras, and P. B. Wilkinson, "Recent developments in the direct-current geoelectrical imaging method," Journal of Applied Geophysics, vol. 95, pp. 135-156, 2013.

[6] A. Samouëlian, I. Cousin, A. Tabbagh, A. Bruand, and G. Richard, "Electrical resistivity survey in soil science: a review," Soil and Tillage Research, vol. 83, no. 2, pp. 173-193, 2005. 
[7] V. M. Timofeev, A. W. Rogozinski, and J. A. Hunter, "A new ground resistivity method for engineering and environmental geophysics," in Proceedings of the Symposium on the Application of Geophysics to Engineering and Environmental Problems (SAGEEP'94), pp. 701-715, 1994.

[8] O. Kuras, D. Beamish, P. I. Meldrum, and R. D. Ogilvy, "Fundamentals of the capacitive resistivity technique," Geophysics, vol. 71, no. 3, pp. G135-G152, 2006.

[9] M. Neukirch and N. Klitzsch, "Inverting capacitive resistivity (line electrode) measurements with direct current inversion programs," Vadose Zone Journal, vol. 9, no. 4, pp. 882-892, 2010.

[10] O. Kuras, The capacitive resistivity technique for electrical imaging of the shallow subsurface [Ph.D. thesis], University of Nottingham, 2002.

[11] Geometrics, Ohmmapper, http://www.geomatrix.co.uk/landproducts/electrical-resistivity/ohmmapper/.

[12] IRIS Instruments, "Corim shallow resistivity," http://www.irisinstruments.com/Pdf file/Corim_Gb.pdf.

[13] D. Groom, "Common misconceptions about capacitivelycoupled resistivity (CCR) what it is and how it works," in Proceedings of the 21st EEGS Symposium on the Application of Geophysics to Engineering and Environmental Problems (SAGEEP '08), Philadelphia, Pa, USA, April 2008.

[14] G. A. Oldenborger and A.-M. LeBlanc, "Capacitive resistivity inversion using effective dipole lengths for line antennas," Journal of Applied Geophysics, vol. 98, pp. 229-236, 2013.

[15] Q. Niu and Y.-H. Wang, "Theoretical and experimental examinations of the capacitively coupled resistivity (line antenna) method," Geophysics, vol. 78, no. 4, pp. E189-E199, 2013.

[16] Q. Niu, Y.-H. Wang, and K. Zhao, "Evaluation of the capacitively coupled resistivity (line antenna) method for the characterization of vadose zone dynamics," Journal of Applied Geophysics, vol. 106, pp. 119-127, 2014.

[17] A. Hibbs, T. Petrov, J. Pendleton, S. Milberger, G. Eiskamp, and G. A. Wilson, "New electromagnetic sensors for magnetotelluric and induced polarization geophysical surveys," SEG Technical Program Expanded Abstracts, pp. 1-5, 2012.

[18] A. F. Marsala, A. D. Hibbs, T. R. Petrov, and J. M. Pendleton, "Six-component tensor of the surface electromagnetic field produced by a borehole source recorded by innovative capacitive sensors," in Proceedings of the SEG Annual Meeting, pp. 825829, Society of Exploration Geophysicists, Houston, Tex, USA, September 2013.

[19] J. P. Walker, Estimating soil moisture profile dynamics from nearsurface soil moisture measurements and standard meteorological data [Ph.D. thesis], The University of Newcastle, 1999.

[20] P. Rasmussen and R. T. Kinnerup, "Ultra low frequency infrasonic measurement system," Tech. Rep. DK-2800, Technical University of Denmark (DTU), Kongens Lyngby, Denmark, 2011. 


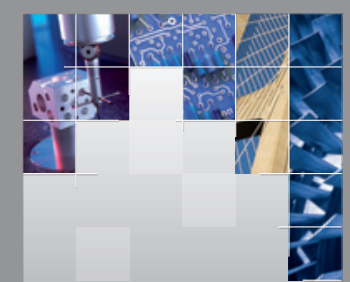

\section{Enfincering}
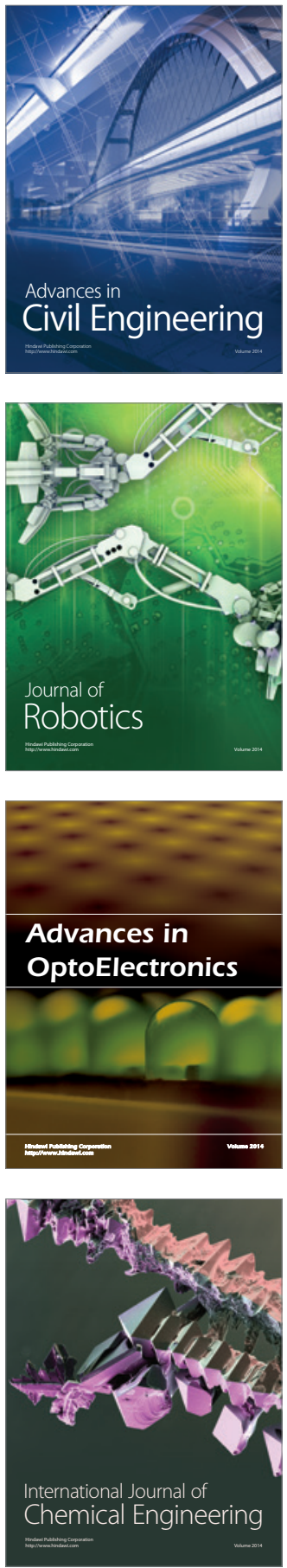

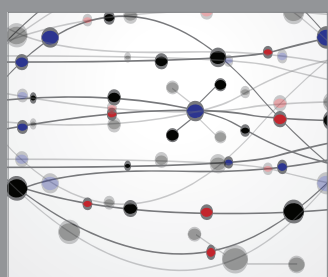

The Scientific World Journal

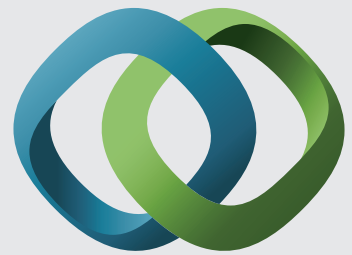

\section{Hindawi}

Submit your manuscripts at

http://www.hindawi.com
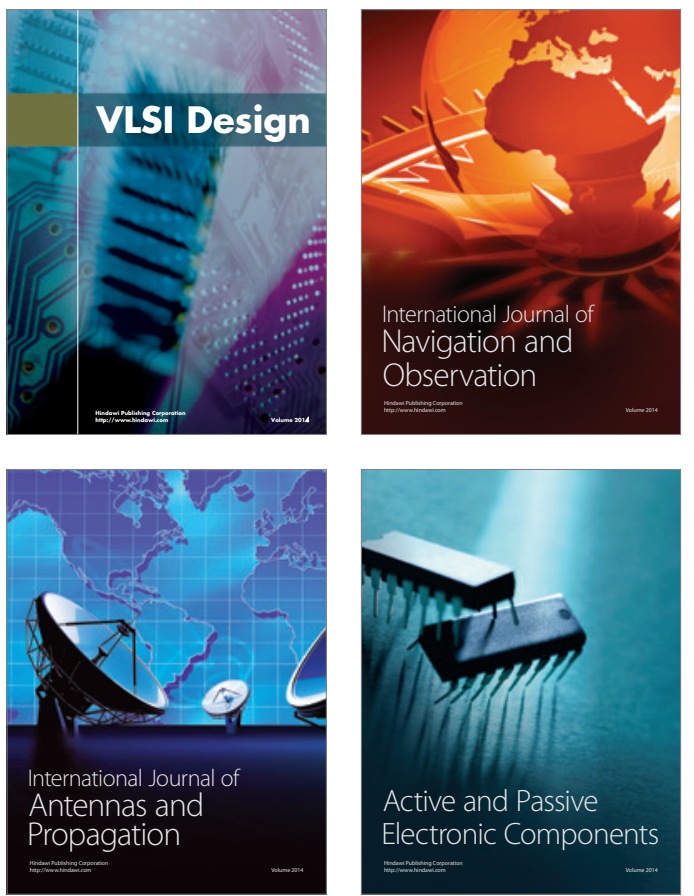
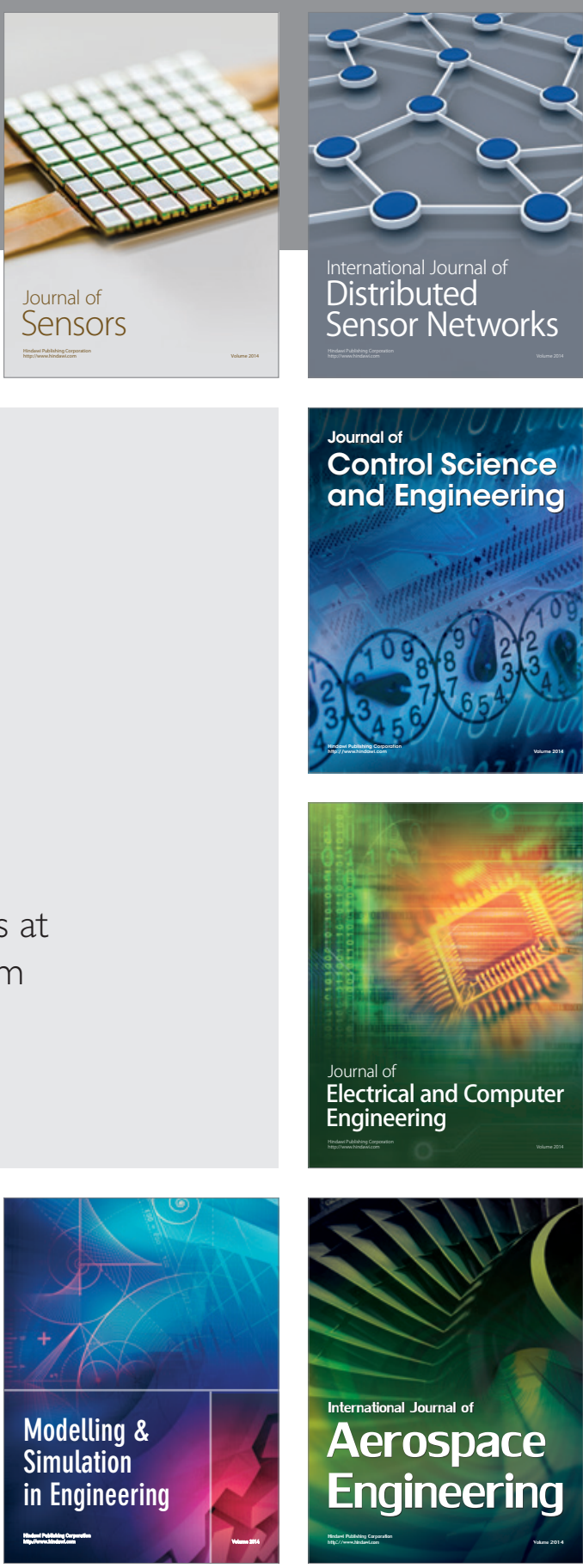

International Journal of

Distributed

Sensor Networks

Journal of

Control Science

and Engineering
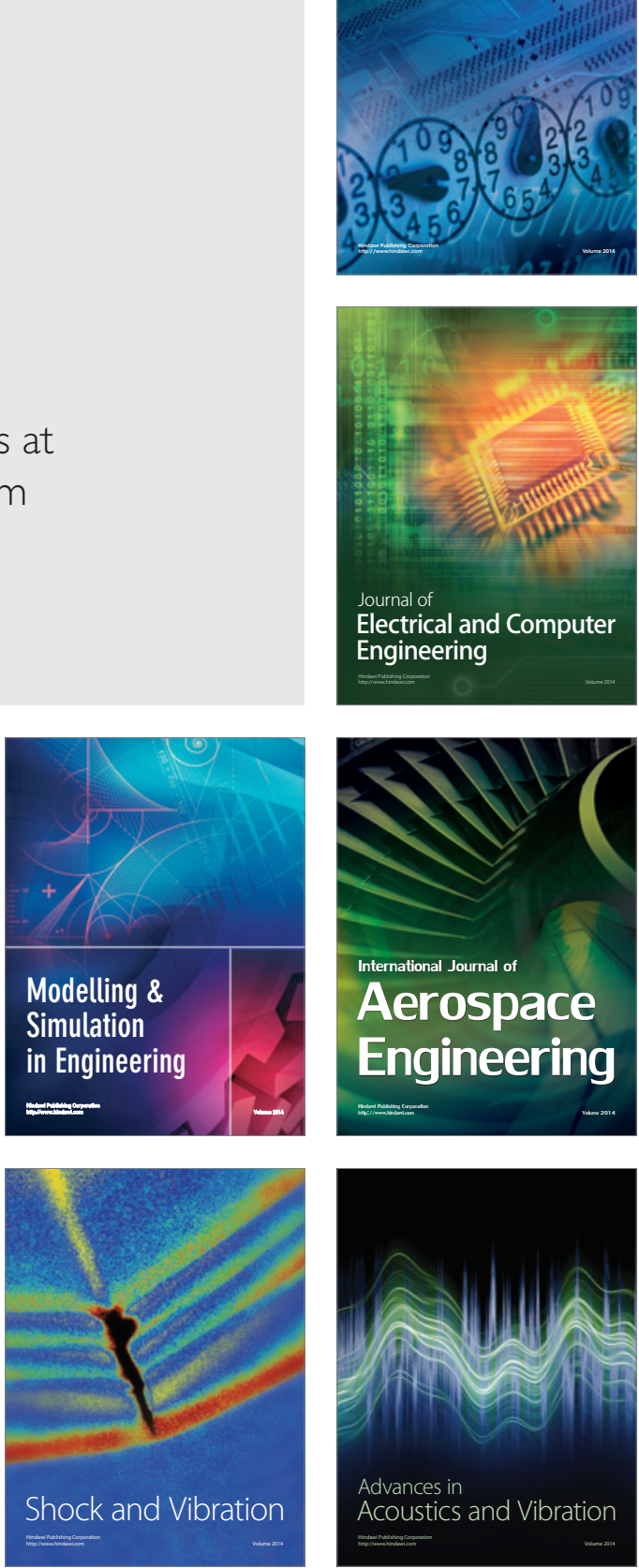